\title{
Metastasis-suppressing NID2, an epigenetically-silenced gene, in the pathogenesis of nasopharyngeal carcinoma and esophageal squamous cell carcinoma
}

\author{
Annie Wai Yeeng Chai ${ }^{1}$, Arthur Kwok Leung Cheung ${ }^{1}$, Wei Dai ${ }^{1}$, Josephine Mun \\ Yee Ko ${ }^{1}$, Joseph Chok Yan Ip ${ }^{1}$, Kwok Wah Chan ${ }^{2,3}$, Dora Lai-Wan Kwong ${ }^{1,4}$, Wai \\ Tong Ng ${ }^{4,5}$, Anne Wing Mui Lee ${ }^{1,4}$, Roger Kai Cheong Ngan ${ }^{4,6}$, Chun Chung $\mathrm{Yau}^{4,7}$, \\ Stewart Yuk Tung ${ }^{4,8}$, Victor Ho Fun Lee ${ }^{1,4}$, Alfred King-Yin Lam ${ }^{9}$, Suja Pillai ${ }^{9}$, Simon \\ Law $^{2,10}$, Maria Li Lung ${ }^{1,2,4}$ \\ ${ }^{1}$ Department of Clinical Oncology, The University of Hong Kong, Hong Kong (SAR), People's Republic of China \\ ${ }^{2}$ Center for Cancer Research, The University of Hong Kong, Hong Kong (SAR), People's Republic of China \\ ${ }^{3}$ Department of Pathology, The University of Hong Kong, Hong Kong (SAR), People's Republic of China \\ ${ }^{4}$ Center for Nasopharyngeal Carcinoma Research, The University of Hong Kong, Hong Kong (SAR), People's Republic of China \\ ${ }^{5}$ Department of Clinical Oncology, Pamela Youde Nethersole Eastern Hospital, Hong Kong (SAR), People's Republic of China \\ ${ }^{6}$ Department of Clinical Oncology, Queen Elizabeth Hospital, Hong Kong (SAR), People's Republic of China \\ ${ }^{7}$ Department of Oncology, Princess Margaret Hospital, Hong Kong (SAR), People's Republic of China \\ ${ }^{8}$ Department of Clinical Oncology, Tuen Mun Hospital, Hong Kong (SAR), People's Republic of China \\ ${ }^{9}$ Department of Cancer Molecular Pathology, Griffith Medical School and Menzies Health Institute Queensland, Griffith \\ University, Gold Coast, Australia \\ ${ }^{10}$ Department of Surgery, The University of Hong Kong, Hong Kong (SAR), People's Republic of China
}

Correspondence to: Maria Li Lung, email: mlilung@hku.hk

Keywords: Nidogen-2 (NID2), promoter hypermethylation, metastasis, nasopharyngeal carcinoma (NPC), esophageal squamous cell carcinoma (ESCC)

Received: June 21, $2016 \quad$ Accepted: October 19, $2016 \quad$ Published: October 25, 2016

\section{ABSTRACT}

Nidogen-2 (NID2) is a key component of the basement membrane that stabilizes the extracellular matrix (ECM) network. The aim of the study is to analyze the functional roles of NID2 in the pathogenesis of nasopharyngeal carcinoma (NPC) and esophageal squamous cell carcinoma (ESCC). We performed genome-wide methylation profiling of NPC and ESCC and validated our findings using the methylation-sensitive high-resolution melting (MS-HRM) assay. Results showed that promoter methylation of NID2 was significantly higher in NPC and ESCC samples than in their adjacent non-cancer counterparts. Consistently, down-regulation of NID2 was observed in the clinical samples and cell lines of both NPC and ESCC. Re-expression of NID2 suppresses clonogenic survival and migration abilities of transduced NPC and ESCC cells. We showed that NID2 significantly inhibits liver metastasis. Mechanistic studies of signaling pathways also confirm that NID2 suppresses the EGFR/Akt and integrin/ FAK/PLCY metastasis-related pathways. This study provides novel insights into the crucial tumor metastasis suppression roles of NID2 in cancers.

\section{INTRODUCTION}

Cancer is a multifactorial disease arising as a consequence of both genetic and epigenetic alterations [1]. In recent decades, there has been intensified focus on the study of cancer epigenetics, with the aim to better understand the disease and develop novel therapeutics targeting epigenetic changes. Aberrant promoter hypermethylation of tumor suppressor genes (TSGs) and/ or metastasis suppressor genes (MSGs) has been well- 
studied and is known to be a key driver of tumorigenesis in several cancers [2-4].

Nidogen-2 (NID2) encodes a secretory protein from the nidogen protein family [5]. The NID2 and also its family member, NID1, are ubiquitously present in the basement membrane and serve to maintain the integrity and stability of the basement membrane by connecting laminin and collagen IV networks in the extracellular matrix (ECM) [5, 6]. NID2 silencing was reported in many cancer types and the aberrant promoter hypermethylation is one of the most critical events detected in different malignancies, including gastric [7], bladder [8, 9], and invasive cervical [10] cancers. Detection of NID2 methylation was proposed as a biomarker for the diagnosis of many cancers including non-small cell carcinoma of lung [11], urothelial carcinoma of urinary bladder [9, $12,13]$ and squamous cell carcinoma of oral cavity [14]. Furthermore, a functional study revealed that NID2deficient mice have higher lung metastasis upon tail vein injection of melanoma cells [15], suggesting a critical role of NID2 in suppressing the metastatic potential of cancer cells.

Previously, we utilized the Illumina HumanMethylation450 Bead Chip (HM450) to examine the methylome profile of two cancers of the aerodigestive tract of relatively high prevalence in China, namely, nasopharyngeal carcinoma (NPC) [16] and esophageal squamous cell carcinoma (ESCC). A number of TSGs silenced by means of promoter hypermethylation has already been identified in these two cancers [17-23]. They are confirmed by our previous functional genetics and epigenetics approaches. From the methylome analyses, NID2 was confirmed to be the top hit as a promoter hypermethylated gene in both NPC and ESCC. As NID2 harbors several de novo methylated loci in the $\mathrm{CpG}$ islands, it is a potential TSG/MSG in these cancers.

Despite the substantial number of studies associating NID2 to different cancers, to the best of our knowledge, there have not been any in-depth functional studies to elucidate the potential suppressive role of NID2 in cancers, especially in NPC and ESCC. Hence, in the present study, we aimed to scrutinize the functional role of NID2 in these cancers.

\section{RESULTS}

\section{Down-regulation of NID2 is highly associated with aberrant promoter hypermethylation in both NPC and ESCC}

Our previous HM450 methylome analysis of 25 primary NPC [16] and 17 primary ESCC (unpublished data) and their matched adjacent non-cancer tissues has shown that hypermethylation is important in these cancers. This is a frequent event in NPC compared to many other cancer types [24]. In this study, we analyzed our NPC and
ESCC methylome data to identify candidate genes that are regulated by aberrant methylation. Among the genes that showed differential methylation, NID2 was one of the top candidate genes showing significant differences in the methylation levels between cancer and noncancer specimens. In both NPC and ESCC, the CpG-rich promoter regions of NID2 were hypermethylated, when compared to the matched non-cancer tissues (Figure 1A) (Supplementary Figure S1).

In addition to global HM450 methylome studies, our previous functional complementation studies utilized the microcell-mediated chromosome transfer (MMCT) approach to transfer an intact human chromosome 14 into the tumorigenic HONE1 cell line for identification of TSGs [18]. A panel of tumor-suppressive microcell hybrids (MCHs) and tumorigenic tumor segregants (TSs) was established. Studies found that TSGs usually show up-regulation in the MCHs, while being down-regulated in the TSs [18]. NID2 showed significant up-regulation in the MCHs and down-regulation in the matched TSs (Figure 1B). This functional complementation study further supported the potential of the NID2 to function as a tumor suppressor or metastasis suppressor.

To evaluate the NID2 promoter hypermethylation in NPC and ESCC, we identified the region that showed distinct differential methylation in selected NPC cell lines, when compared to the NP cell lines (Supplementary Figure S2). The high-throughput methylation-sensitive high-resolution melting (MS-HRM) assay was used to assess the promoter methylation status of NID2 in clinical samples. We found that $74 \%$ of 50 NPC biopsies had a methylated NID2 promoter, while the majority of the adjacent non-cancer tissues remained unmethylated $(66 \%)$ (Figure 2A). In ESCC, a trend of NID2 promoter hypermethylation was detected in $80 \%$ of the samples (Figure 2A), which was consistent with our unpublished ESCC methylome data using the same samples. These MS-HRM results further validate the clinical significance of the NID2 promoter hypermethylation, supporting our finding from the HM450. The MS-HRM results showed that the NID2 promoter is methylated in HONE1, HK1, and C666 (NPC cell lines), when compared to the unmethylated immortalized nasopharyngeal cell lines (NP69 and NP460). Similarly, the NID2 promoter is also methylated in the ESCC cell lines (KYSE30, KYSE150, and SLMT-1S1), while the NID2 promoter in NE3 and NE083 (immortalized esophageal cell lines) remain unmethylated (Supplementary Figure S3).

The expression levels of NID2 were determined in paired NPC/ESCC and non-cancer biopsies to validate their clinical relevance. NPC [50\% (37/74)] and ESCC biopsies $[43 \%(12 / 28)]$ showed more than two-fold down-regulation of NID2 (Figure 2B). These data support NID2 being a potential TSG in ESCC and NPC. Immunohistochemical (IHC) analysis of NID2 was used to show protein expression levels directly in cancer tissues. 
Representative images are shown in Supplementary Figure $\mathrm{S} 4$, in which stronger staining in the non-neoplastic glands of nasopharyngeal mucosa was observed in comparison to the staining in NPC. qPCR analysis in the cell lines showed that NID2 is down-regulated in $100 \%(7 / 7)$ of NPC cell lines and 80\% (12/15) of ESCC cell lines (Figure 2C). Consistent with the MS-HRM results, NID2 expression was lower in cell lines that had methylated NID2 promoter (HONE1, HK1, C666-1, KYSE30, KYSE150, and SLMT-1S1), when compared to the respective non-neoplastic immortalized cell lines with an unmethylated NID2 promoter.

\section{NID2 re-expression suppresses NPC and ESCC cancer-associated characteristics: Clonogenic survival and cancer cell migration and invasion abilities}

To confirm the effect of NID2 on both NPC and ESCC disease progression, NID2 was re-expressed in NPC (HONE1) and ESCC (KYSE30) cells. Detectable NID2 protein was found in both cell lysates and conditioned media (Figure 3A). The NID2-expressing cells were then used for the downstream functional studies.
The clonogenic survival ability of tumor cells is a prerequisite for occurrence of metastasis and was assessed by 2D and 3D colony formation assays. NID2expressing HONE1 cells significantly reduced colonyforming ability. The number of colonies formed was only $43.4 \%$ of the vector-alone (VA) control. KYSE30 cells expressing NID2 only retained $38 \%$ of the colonyforming ability (Figure 3B). The NID2-expressing cells were also cultured on the 3D Matrigel matrix, to mimic the ECM environment. They showed significant reduction of clonogenic survival ability. On average, the number of tumor spheres $(\geq 100 \mu \mathrm{m})$ formed in NID2-expressing HONE1 and KYSE30 was reduced to $62 \%$ and $45 \%$, respectively, of the VA control (100\%) (Figure 3C).

Previous study suggested that NID2-deficient mice have higher lung metastasis [15], implying a critical role of NID2 in suppressing cell motility and, thus, metastasis. We examined the ability of NID2 to inhibit in vitro migration in the wound healing assay. NID2 re-expression in both HONE1 and KYSE30 impaired migration ability, as seen by the delayed wound closure, when compared to VA control (Figure 4A). In the in vitro migration chamber assay, the relative migration abilities were also significantly lower in NID2-expressing HONE1 (68\%,

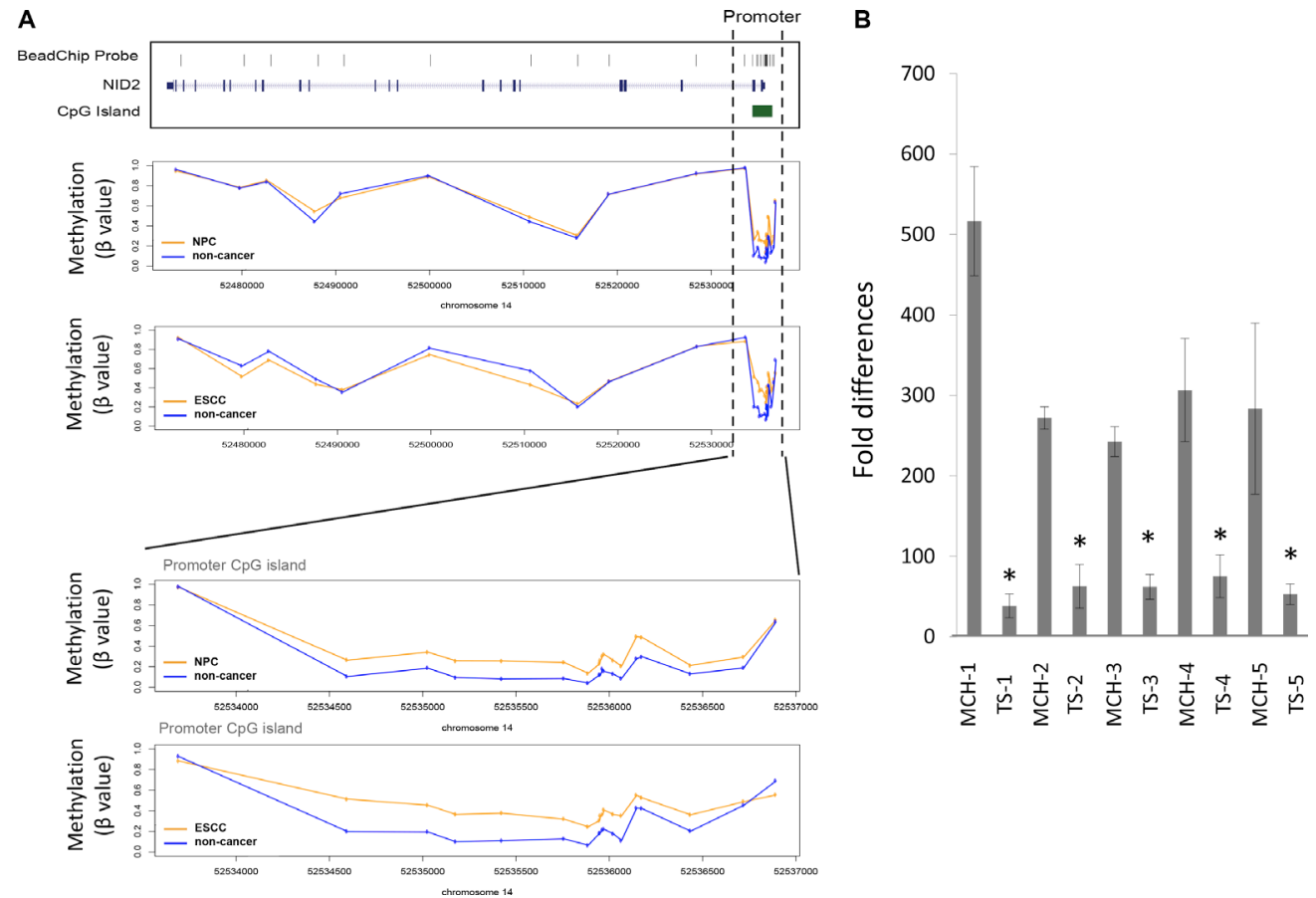

Figure 1: NID2 is identified as a candidate gene in NPC and ESCC. (A) The average methylation level of NID2 derived from our previous methylome data in NPC and ESCC. The vertical broken line shows the region covering the promoter CpG island (chr14: 52534582-52536722) and the bottom figures show a close-up view of changes in methylation. Methylation level is presented as $\beta$ value ( $\beta$ $=\mathrm{M} /(\mathrm{U}+\mathrm{M}+100)$, $\mathrm{M}$ : signal intensity of the methylated allele, $\mathrm{U}$ : signal intensity of the unmethylated allele). The y-axis shows the average methylation level in tumors (orange line) and non-cancer controls (blue line), respectively. Within this region, the methylation levels in multiple CpG sites of both NPC and ESCC patients are consistently higher than those of non-cancer controls, with adjusted $p$ value $<0.05$ estimated by LIMMA analysis using the transformed $\beta$ values as previously described [16]. Significance level of each selected probes were shown in Supplementary Figure S 1. (B) MMCT of chromosome 14 was previously performed using HONE1 as the recipient cell line [18]. qPCR analysis of tumor-suppressive microcell hybrids (MCHs) and their tumor segregant (TS) cell lines, which are no longer tumorsuppressive, showed that NID2 expression was down-regulated in all five TS cell lines, when compared to their respective MCHs. Asterisk (*) indicates samples with more than two-fold differences compared to its MCHs. 
$* p<0.05)$ and $\operatorname{KYSE} 30\left(56 \%,{ }^{*} p<0.05\right)$ (Figure 4B). In Matrigel-coated invasion chambers, re-expression of NID2 led to significant reduction in the relative invasiveness of HONE1 $(67 \%, * p<0.05)$ and KYSE30 $(58 \%, * * p<0.01)$ (Figure 4B). These results confirmed the functional role of NID2 in suppression of in vitro cancer cell migration and invasion.

\section{NID2 negatively regulates the cancer-related EGFR/Akt and Integrin/FAK/PLC $\gamma$ pathways associated with ECM protein signaling}

Studies of how NID2 may be involved in different cancer signaling pathways are still lacking. The Human Phospho-Kinase Antibody Array was utilized to explore the possible cancer signaling pathways that may be affected by NID2. NID2 re-expression significantly reduced the phosphorylation levels of several cancerrelated molecules in the key ECM protein-related pathways, including FAK (Y397) and PLC $\gamma$ (Y783) of the integrin signalling pathways and Akt (T308) of the epidermal growth factor receptor (EGFR) downstream signaling pathways (Figure 5A).

Cell-ECM interactions are crucial in regulating the behavior of cells. The signal transduction cascade induced by alteration in the ECM is mediated by integrins

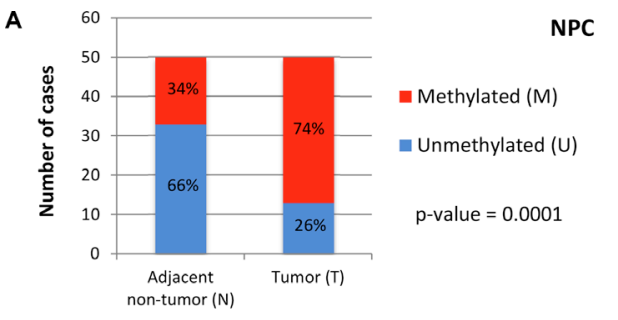

B
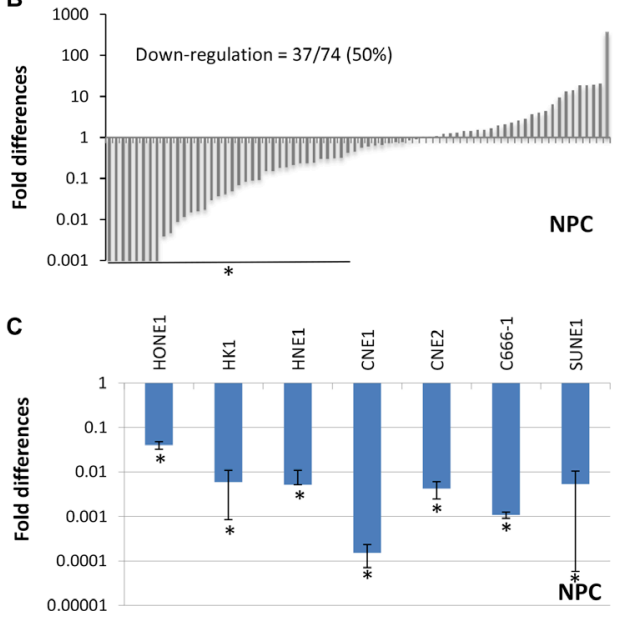

and EGFR. Members of the integrin-mediated ECM signaling pathways such as FAK and PLC $\gamma$ have reduced phosphorylation upon NID2 expression, suggesting an overall inactivation of integrin signaling (Figure 5B). Western blot analyses on NID2-expressing cells validate our findings in the human phosphorylation array. Previous study suggested that the inhibition of EGFR/Akt signalling significantly suppresses cancer cell metastasis, including NPC metastasis [25]. NID2 re-expression in cancer cells revealed attenuated EGFR activity. There were reduced levels of the phosphorylated form of EGFR at various tyrosine sites (Y992, Y1068, Y1086, Y1148, and Y1173) (Figure 5C). NID2 also inhibited the downstream Akt pathway. Reduction in phospho-Akt levels was observed at T308 and Y473 sites (Figure 5C). These results support the crucial role for NID2 in the suppression of these cancer-associated signaling pathways.

\section{NID2 potently inhibits in vivo metastasis, but does not affect in vivo subcutaneous tumor growth}

Previous study showed that NID2-deficient mice have a higher lung metastasis frequency, when intravenously injected with melanoma cells [15]. In this study, the functional role of NID2 in the suppression of
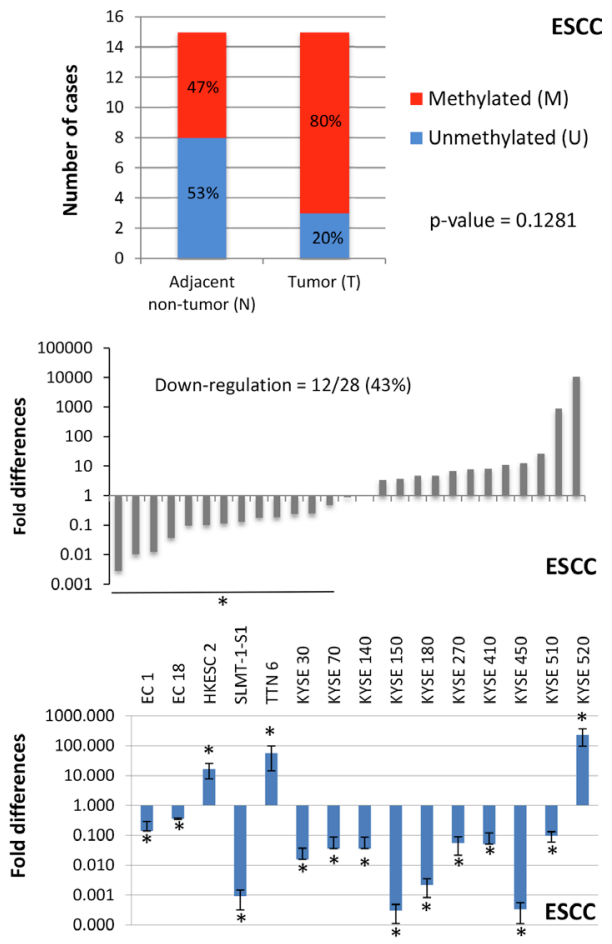

Figure 2: Validation of NID2 promoter hypermethylation and its down-regulation in NPC and ESCC. (A) In 37 of 50 $(74.0 \%, p$-value $=0.0001)$ NPC biopsies, the NID2 promoter hypermethylation was observed. The NID2 promoter was methylated in 12 of $15(80.0 \%, p$-value $=0.1281)$ ESCC biopsies, as shown by MS-HRM results. (B) qPCR analysis showed that expression of NID2 is down-regulated in 50\% (37/74) of NPC biopsies and 43\% (12/28) of ESCC biopsies showing more than two-fold down-regulation of NID2 expression. (C) All 7 NPC cell lines showed down-regulation of NID2 expression, after normalization to the immortalized nasopharyngeal cell line. $75 \%$ of the ESCC cell lines (12 of 16) have decreased NID2 expression, when compared to the immortalized normal esophageal epithelial cell line. The housekeeping gene $G A P D H$ was used as an internal control for all qPCR analysis and the asterisks $(*)$ indicate samples with more than two-fold up-regulation $(>2.0)$ or down-regulation $(<0.5)$. 


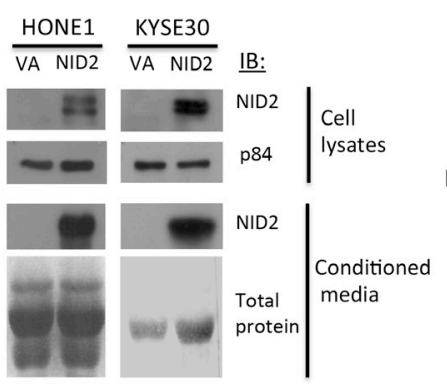

C

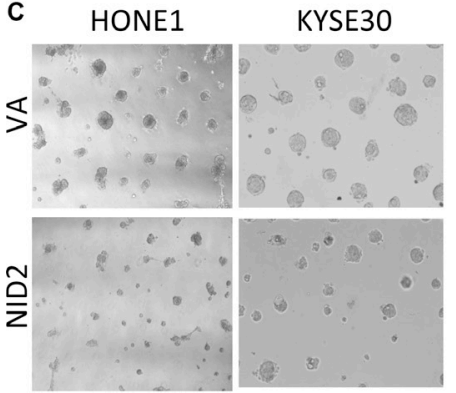

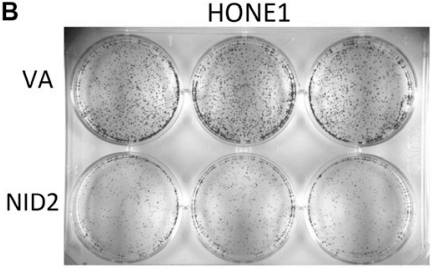
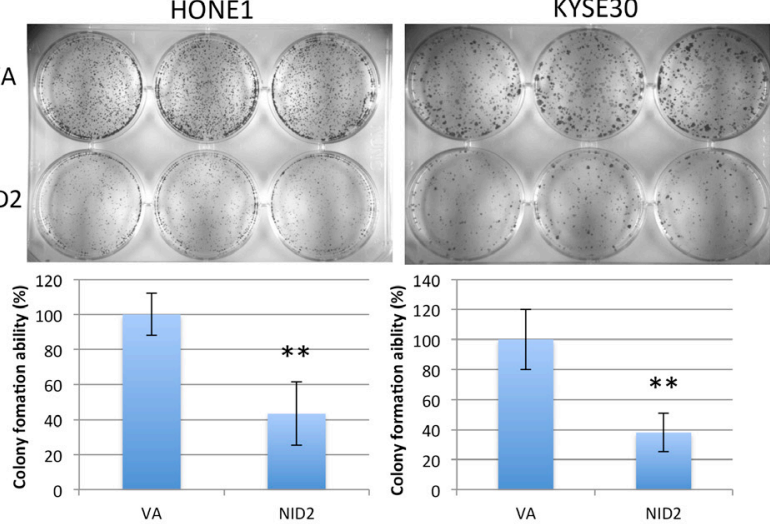
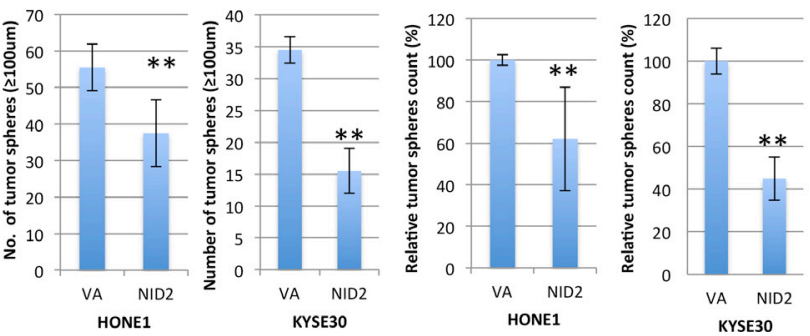

Figure 3: NID2 inhibits 2D and 3D clonogenic survival. (A) Western blot analysis confirmed re-expression of NID2 in selected NPC (HONE1) and ESCC (KYSE30) cell lines, which have down-regulated NID2 expression. Over-expression of NID2 was detected in cell lysates, as well as being secreted into the conditioned media. Coomassie blue staining of total protein was used as a loading control for the conditioned media. (B) 2D colony formation assays of HONE1 and KYSE30 cells. Re-expression of NID2 significantly reduced their colony-forming abilities to around $40 \%(* * p<0.01)$. Data shown are the mean from three independent experiments \pm S.D. (C) In 3D Matrigel culture, cells re-expressing NID2 resulted in significantly lower numbers of tumor spheres $(\geq 100 \mu \mathrm{m})$, when compared to the VA in both NID2-expressing HONE1 and KYSE30 cells $(* * p<0.01)$. Data shown are the mean from three independent experiments \pm S.D.

A

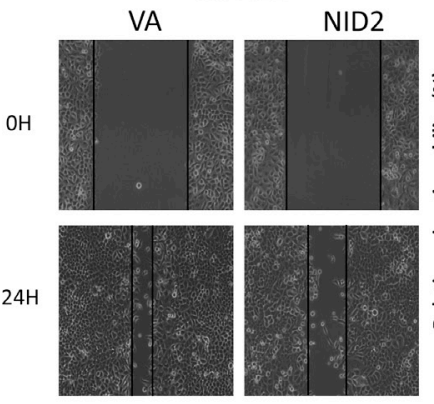

KYSE30

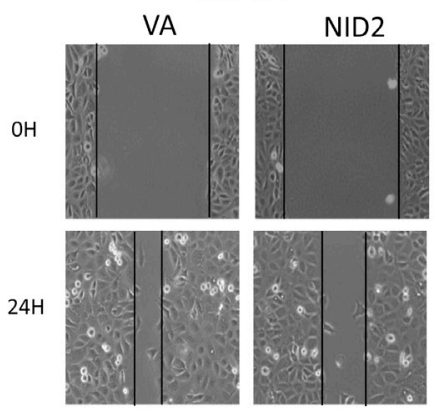

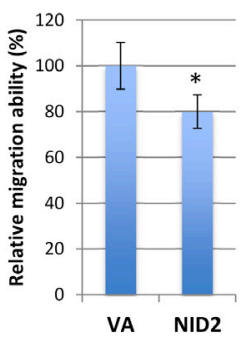

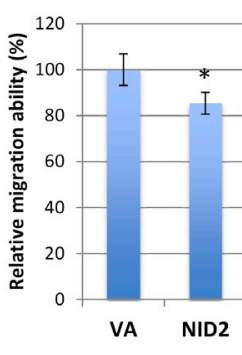

B

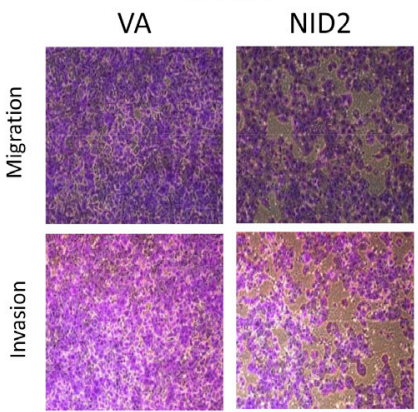

KYSE30

VA

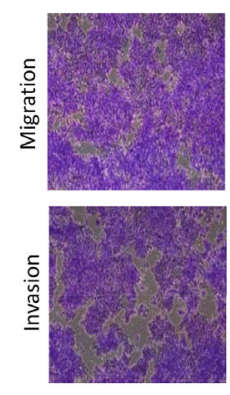

NID2

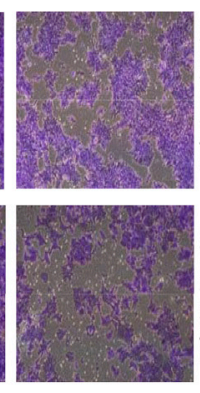

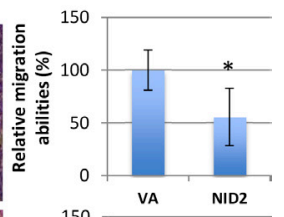
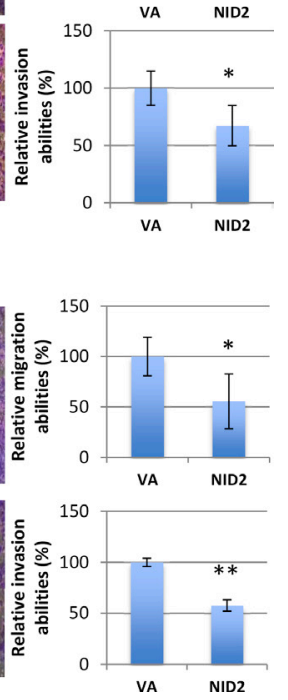

Figure 4: NID2 suppresses cell migration and invasion. (A) Cell migration ability was evaluated by the wound healing assay. Microscopic images were taken at 0th hour and 24th hour to monitor the extent of wound closure. Both NID2-expressing HONE1 and KYSE30 cells showed impaired wound healing ability, when compared to the VA controls $\left({ }^{*} p<0.05\right)$. (B) Representative fields of migrated or invaded cells at 100x magnification. Re-expression of NID2 resulted in significant reduction of migration and invasion abilities of HONE1 and KYSE30 cells $\left(* p<0.05,{ }^{* *} p<0.01\right)$. Data shown are the mean from three independent experiments \pm S.D. 
in vivo metastasis was examined by intrasplenic injection of metastatic cancer cells. The NID2-expressing cells and the VA control were used for intrasplenic injection into nude mice [25] and the in vivo metastasis status was monitored weekly by the Xenogen live animal imaging system (Figure 6A). The nude mice were euthanized at week 3 post-injection, followed by necropsy. Using the highly metastatic HONE1-Luc cells, tumor nodules were clearly visible in $90 \%$ of the livers from the VA group (Figure 6B). The mice from the VA group showed a high metastasis rate to the liver (10 of 11 mice; $90 \%$ ), whereas in the NID2 group, there were no metastasized tumors observed in the excised livers (0 of 12 mice; $0 \%$; $p$-value $=0.0001)($ Figure $6 \mathrm{C})$. The results were further validated by $H \& E$ staining. Representative $H \& E$ staining images of metastatic liver tumor nodules and metastasisnegative samples are shown (Figure 6D). These results provide strong evidence for a functional role of NID2 in the inhibition of the in vivo metastasis.

In order to eliminate the possibility that the difference in the ability for metastasis is induced by the inhibition of the in vivo tumor growth by NID2, the

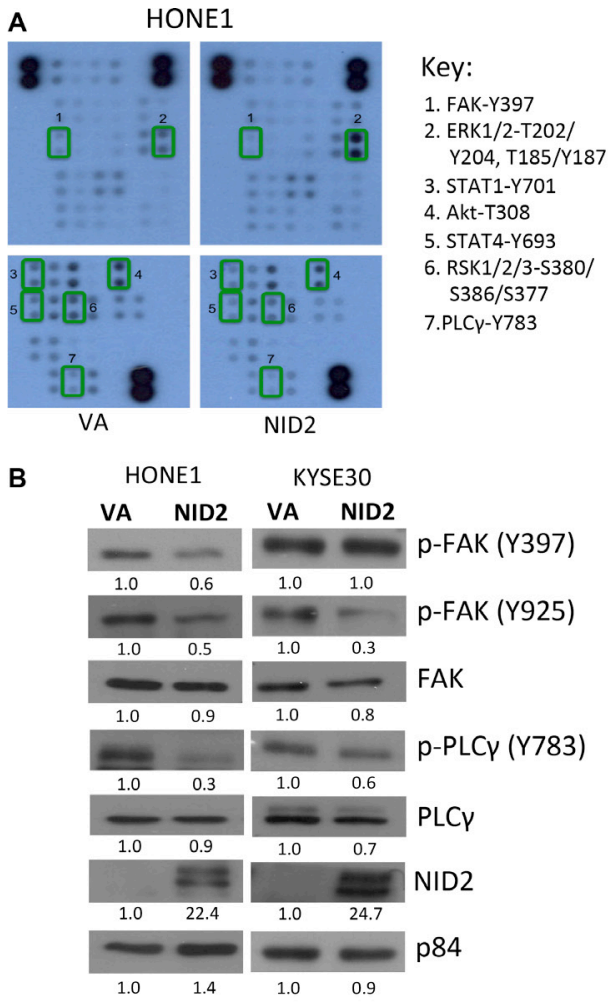

in vitro cell proliferation assay and in vivo subcutaneous nude mouse tumorigenicity assay were performed. Results suggested that NID2 re-expression was unable to induce significant changes in both the in vitro cell proliferation and in vivo tumorigenicity of the cancer cells (Figure $7 \mathrm{~A}$ and $7 \mathrm{~B}$ ). These results provide further evidence in support of NID2 playing an important role in metastasis suppression, despite it not affecting primary tumor growth.

\section{DISCUSSION}

In this study, we examined the methylome of NPC and ESCC tumors and successfully identified a hypermethylated gene, NID2, which is a potential TSG/ MSG. This possibility was further supported by our previous chromosome 14 MMCT NPC study. Frequent allelic deletions of chromosome $14 \mathrm{q}$ have also been observed in both NPC [26] and ESCC [27], where NID2 gene is mapped. NID2 was also consistently found to be hypermethylated in several other cancers [7-14, 28-30].

NID2 is an ECM protein and an important member of the basement membrane. Deregulated ECM signaling
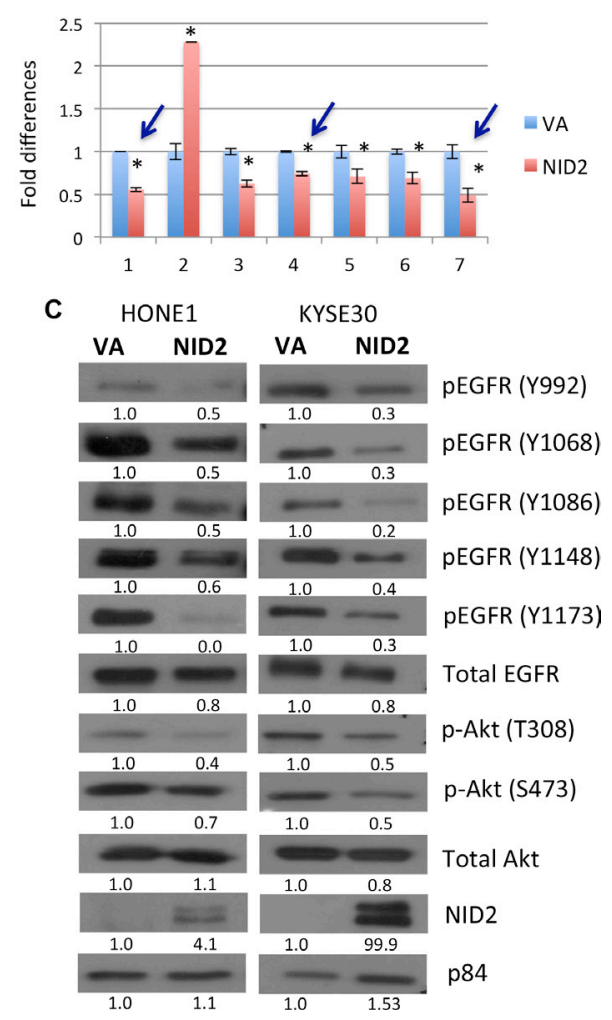

Figure 5: NID2 suppresses important signaling pathways in cancer. (A) Phosphorylation study of NID2-expressing cells using the Human Phospho-Kinase Antibody Array. Reduced phosphorylation of (1) FAK (Y397), (3) STAT1 (Y701), (4) Akt (T308), (5) STAT4 (Y693), (6) RSK1/2/3 (S380/S386/S377), and (7) PLC $\gamma$ (Y783), and increased phosphorylation of (2) ERK1/2 (T202/Y204, T185/Y187) in the NID2-expressing cells was observed. The bar chart shows the relative quantification of the array data, normalized to VA control $(* p$ $<0.05$ ). Blue arrows indicated significant changes that were further validated using Western blot. (B) Re-expression of NID2 in HONE1 and KYSE30 cell lines resulted in reduced phosphorylation levels of FAK (Y397/Y925) and PLC $\gamma$ (Y783), as shown by representative Western blot images. (C) The levels of phosphorylated EGFR at five tyrosine sites (Y992/ Y1068/Y1086/Y1148/Y1173) were decreased by the re-expression of NID2 in both HONE1 and KYSE30. Downstream Akt pathways were also suppressed by NID2, as shown by the reduced phosphorylation level of Akt (T308/S473). Numbers at the bottom of each band represent the relative quantification of the protein level, normalized to the VA control. 
A

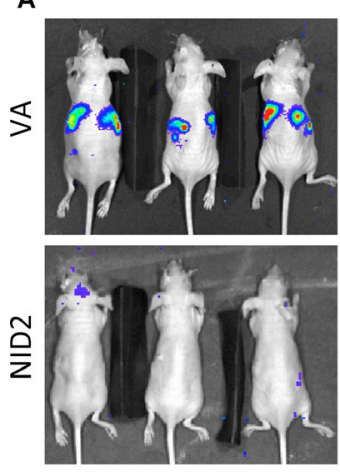

B
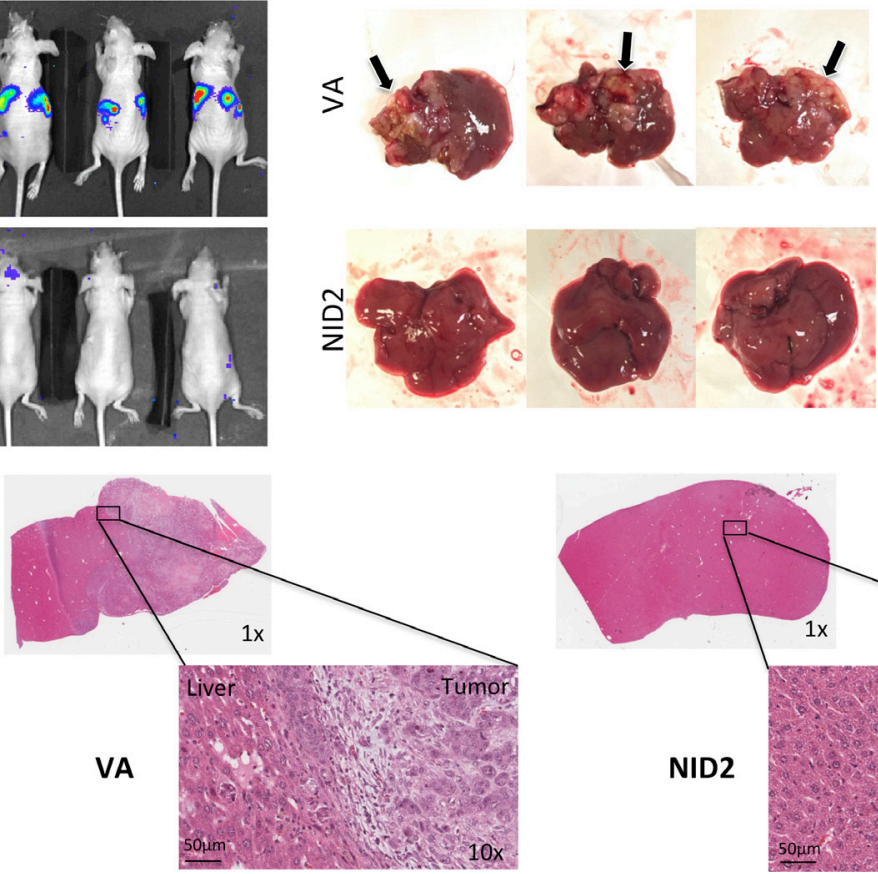

C
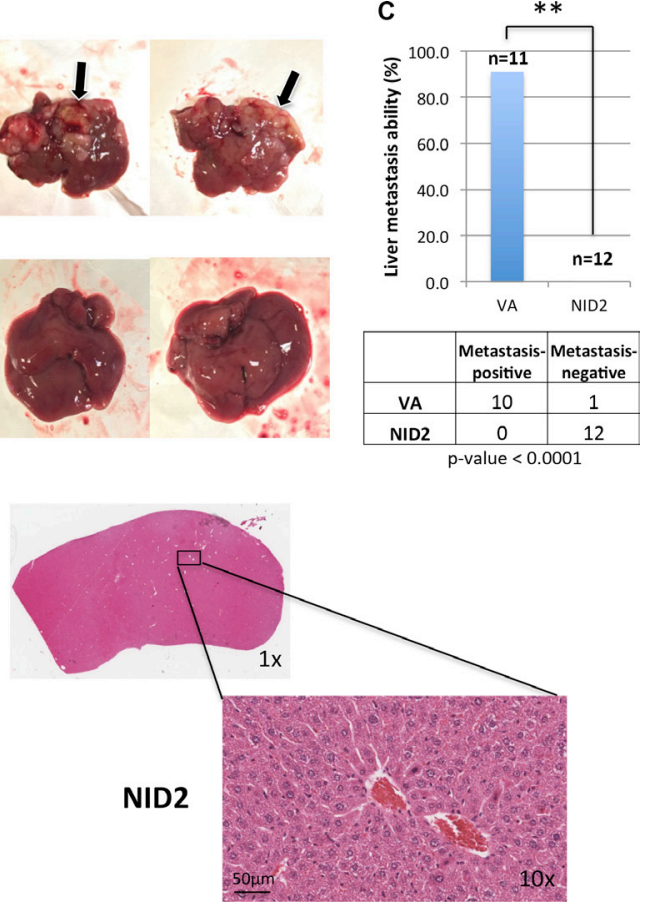

Figure 6: NID2 significantly suppresses cancer metastasis in vivo. (A) Representative images of nude mouse bioluminescent imaging to monitor the extent of in vivo metastasis after intrasplenic injection (B) Lesions on liver were apparent to the naked eye, for those in which metastasis occurred, and are indicated with arrows. None of the excised livers in the NID2 group showed overt tumor lesions. (C) Comparison of in vivo metastasis ability between the two groups revealed a significantly strong suppression of liver metastasis in the NID2 group, in which 0 of 12 mice developed tumors, while in contrast, 10 of 11 mice in the vector control group developed liver metastasis $\left({ }^{* *} p<0.0001\right)$ (D) Histological examination of the excised livers confirms the presence of multiple tumors. Representative H\&E stained images of mouse liver from the VA group with metastatic undifferentiated carcinoma and mouse liver from NID2-expressing group, with no observable tumor.

A

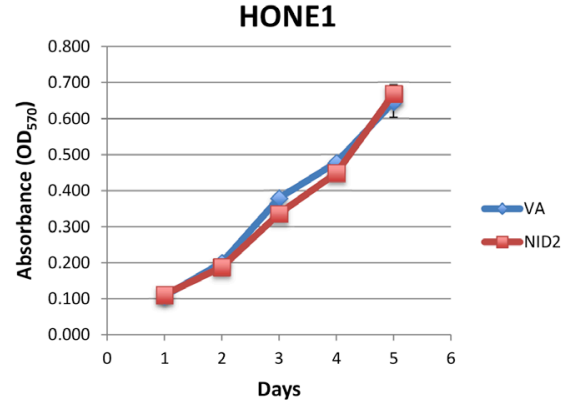

B

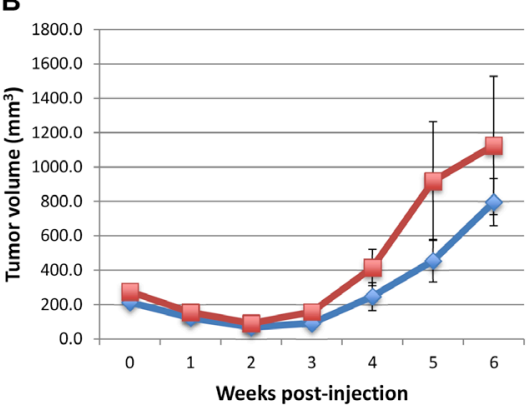

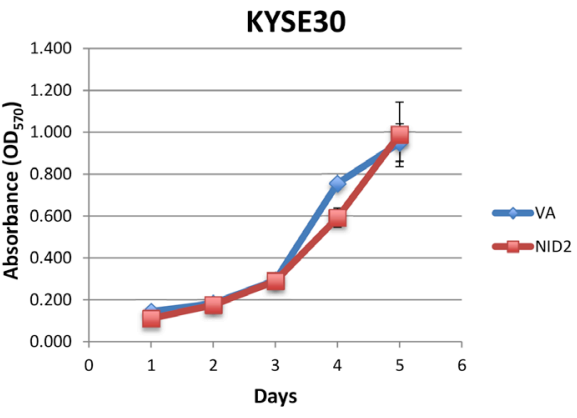

KYSE30

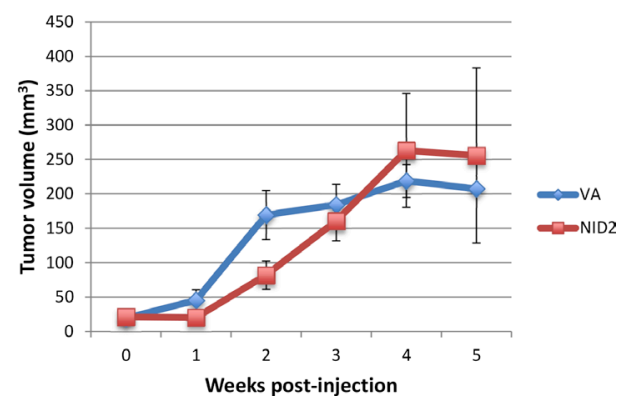

Figure 7: NID2 does not affect cell proliferation in vitro and tumor growth in vivo. (A) In vitro cell proliferation was assessed using the MTT assay. Re-expression of NID2 did not affect HONE1 and KYSE30 cell growth. (B) Nude mouse subcutaneous injection was used to assess the effect of NID2 on in vivo tumor growth. Using both HONE1 and KYSE30, there were no significant differences in the tumor growth kinetics between the NID2-expressing and VA groups. Each data point was plotted as an average tumor volume of six tumor sites \pm S.E.M. 
caused by a disrupted basement membrane network is widely recognized as a hallmark of cancer [31]. The dynamic and complex cell-matrix signal transduction orchestrates cell behavior, which functionally impacts its adhesion, migration, differentiation, and proliferation [32, 33]. Previously, we identified several TSGs in NPC and ESCC that are either encoding ECM proteins ( $F B L N 2$ [21] and LTBP2 $[22,34,35])$ or proteases involved in ECM remodelling (ADAMTS9 [36, 37] and MMP19 [38]). The re-expression of these TSGs was reported to suppress NPC and/or ESCC cell migration, invasiveness, angiogenesis, and in vivo tumor growth [21, 22, 34-36].

The colony formation assay is commonly used to assess the in vitro clonogenic survival ability, which is a critical prerequisite for cancer cells to colonize a secondary site during metastasis $[39,40]$. In this study, concordance with the hypothesis that NID2 is a MSG, NID2 re-expression significantly suppresses the colony formation of both NPC and ESCC cells, in both 2D and 3D Matrigel culture. Furthermore, NID2 also plays a significant role in impairing the migration and invasion abilities of the cancer cells. These in vitro findings support our subsequent in vivo results. NID2-expressing NPC cells significantly inhibit metastasis formation in the liver. Collectively, these results confirm the role of NID2 as a potent suppressor of metastasis in NPC.

Metastasis is a complex process that involves dynamic degradation and remodelling of the ECM. There is increasing recognition of the important role of the ECM-defined tumor microenvironment in tumor initiation and progression. This is exemplified by the discovery of how ECM collagen regulates ECM stiffness and triggers a signaling network to promote breast cancer cell invasion and metastasis [41, 42]. Similarly, Mokkapati et al. reported higher lung metastases for melanoma cells in NID2-null mice [15]. The absence of this important component of the vascular basement membrane facilitated the transmigration of melanoma cells and resulted in a higher metastasis frequency to the lung [15]. This is consistent with findings in this current study, where the re-expression of NID2 significantly inhibited NPC cell metastasis to the liver. The abilities of tumor cells to survive in the blood circulation, to extravasate and to colonize the distant metastatic organ are the key steps governing metastasis, which can all be evaluated in our in vivo metastasis assay. In our study, NID2-expressing cells have markedly lost these abilities and, as a result, none of the mice in the NID2 group had distant metastasis. In contrast, the VA group had a very high percentage of distant metastasis $(90 \%)$, with obvious tumor nodules on the liver. We hypothesize that the tumor microenvironment of NID2-expressing cells in the circulation or liver disfavors the processes of extravasation and colonization during the later cascade of metastasis, leading to the observation of distinctive difference in metastatic outcome, when compared to the control cells. Thus, the down-regulation of NID2 by promoter hypermethylation appears to be a key event leading to cancer metastasis.

As a secretory basement membrane protein constituting the tumor microenvironment, changes in NID2 protein levels can lead to a change in the ECM signaling cascade to redefine cell fate. Integrin molecules are the major receptors mediating extracellular signal transduction into regulating cellular responses such as migration and proliferation [32, 43-45]. It is conventionally believed that upon ECM ligand activation, integrin clusters, and signaling complexes are formed at the focal adhesions, including FAK, Src, PI3K, and PLC $\gamma$ $[46,47]$. Among these, activation of the FAK pathway is known to confer a survival advantage and promote cell motility $[43,47]$. In our study, results from both the Human Phospho-Kinase Antibody Array and Western blot show reduction of phosphorylation in integrin-related downstream molecules such as FAK and PLC $\gamma$, when NID2 is re-expressed. The EGFR/PI3K/Akt pathways are important players in both NPC [25, 48] and ESCC $[49,50]$. Previous study suggested that inhibition of the Akt signalling pathway greatly inhibits in vivo metastasis [25]. Overexpression of EGFR and/or up-regulation of EGFR phosphorylation signaling are common in both cancers [25, 48-50], with Akt playing a central role as the downstream effector molecule [48]. Interestingly, EGFR signaling pathways can also be activated by crosstalk with the integrin pathways, in which they may act synergistically to promote tumorigenesis and metastasis $[51,52]$.

In this current study, we found that restoration of NID2 expression has a negative regulatory role on both EGFR and integrin signaling pathways. We propose that NID2 elicits its in vitro migration/invasion suppression and in vivo metastasis inhibition effects via the negative modulation of these two oncogenic pathways. Further in-depth mechanistic study of how NID2 intervenes with these signaling networks is warranted to further elucidate our understanding of its ability to suppress metastasis. In conclusion, NID2 plays an important role as an ECM protein defining the tumor microenvironment and is involved in metastasis suppression.

\section{MATERIALS AND METHODS}

\section{Cell culture}

All NPC cell lines (HONE1, HK1, CNE1, CNE2, HNE1, SUNE1, and C666) and the immortalized nasopharyngeal epithelial cell lines (NP460 and NP69) were cultured as previously described [53-57]. The HONE1-Luc used for the intrasplenic injection was cultured as previously demonstrated [25]. The panel of tumor-suppressive microcell hybrids (MCHs) and tumorigenic tumor segregants (TSs) was established in an earlier chromosome 14 microcell-mediated chromosome 
transfer study and cultured as previously described [18]. The ESCC cell lines (KYSE30/70/140/150/180/270/ 410/450/510/520, SLMT-1S1, EC1, EC18, HKESC2, and T.Tn6) and the immortalized esophageal epithelial cell lines (NE3 and NE083) were cultured, as detailed $[58,59]$. The 293FT cell line was purchased from Invitrogen (Carlsbad, CA, USA) and cultured as recommended. All cell lines were tested for mycoplasma.

\section{Clinical samples}

For methylation-sensitive high-resolution melting (MS-HRM) analysis, 50 NPC biopsies and matched adjacent non-cancer tissues were collected in Hong Kong between 2010-2013. These specimens were a subset of our previous methylation biomarker study [60]. A total of 17 archived paired ESCC biopsies were obtained from Tuen Mun Hospital and Queen Mary Hospital for the ESCC methylome study (unpublished) and 15 of the informative cases were used in MS-HRM. Specimens utilized for the qPCR were as previously described $[18,59]$.

\section{Extraction and bisulfite conversion of genomic DNA}

Genomic DNA from NPC and ESCC biopsies was extracted using TRIzol (Invitrogen, Carlsbad, CA, USA), as described previously [60]. Genomic DNA was extracted from cell lines using a previously published phenolchloroform extraction protocol [61]. A quantity of $500 \mathrm{ng}$ genomic DNA was used as input for bisulfite conversion [62]. Bisulfite-converted DNA was then precipitated with ethanol and resuspended in $50 \mu \mathrm{l}$ of $10 \mathrm{mM}$ Tris- $\mathrm{HCl}$ (pH 8.5).

\section{Identification of putative NID2 promoter}

Sequences within 200 base pairs of the transcription start site (TSS200) and 5' untranslated region (5'UTR) sequences in the first exon of NID2 were retrieved from the UCSC browser (GRCh37: chr14: 5253548552536146). Putative promoter sequences were identified using PROSCAN (http://www-bimas.cit.nih.gov/molbio/ proscan/) (Supplementary Figure S5). Using MethPrimer [63], a CpG island of 324bp was identified and a pair of primers was designed to amplify a $293 \mathrm{bp}$ region within this island for clonal bisulfite sequencing. The primers used were 5'- TAGTTTGTTGGGTGGGTTTG -3' and 5'CCTTCCTACAAAAACTAATCCCC-3' (Supplementary Figure S6).

\section{Methylation-sensitive high-resolution melting (MS-HRM)}

Primers for MS-HRM were designed based on guidelines from Wodjaz et al. (2008) [64].
An 88bp region containing $12 \mathrm{CpG}$ sites was amplified using the following MS-HRM primers: 5'- CGCGGAGAGTGGGTTGGAGGT - $3^{\prime}$ and 5'ACCACCCGATCCCCCTCCATACT - $3^{\prime}$. MS-HRM analysis was carried out using the Light Cycler 480 System (Roche), as described [60].

\section{Immunohistochemical (IHC) analysis}

IHC staining was performed on NPC biopsies using anti-NID2 (SAB1409003) polyclonal antibody from Sigma-Aldrich (St Louis, MO, USA), following procedures as previously reported [65].

\section{Plasmid construction and lentiviral infection}

The full-length human NID2 gene, in pCEP-PuNID2 vector, was provided by Dr. T Sasaki (FriedrichAlexander-University, Germany). The NID2 open reading frame (ORF) was cloned into a lentiviral expression vector, pLenti6/UbC/V5-DEST (Invitrogen, Carlsbad, CA, USA). The vector-alone (VA) and NID2 constructs were co-transfected with the packaging vector, psPAX2 and the envelope vector, pMD2.G, at a ratio of $4: 3: 1$ $(8 \mu \mathrm{g} / 6 \mu \mathrm{g} / 2 \mu \mathrm{g})$. The human embryonic kidney cell line $293 F T$ was used for virus packaging. Viruses collected 72 hours post-transfection were filtered and stored at $-80^{\circ} \mathrm{C}$ until use. For in vivo study, the NID2 ORF was cloned into the pLVX-EF1 $\alpha$ system and stably transduced cell lines were established.

\section{Quantitative RT-PCR analysis}

Total RNAs were extracted using TRIzol (Invitrogen, Carlsbad, CA, USA). The cDNA was synthesized using M-MLV Reverse Transcriptase (USB, Cleveland, OH, USA) with $1 \mu \mathrm{g}$ of total RNAs. Expression levels of NID2 were measured using quantitative RTPCR with the Applied Biosystems Step-One Plus platform. NID2 and GAPDH Taqman probes were used. Up-regulation is defined as having a fold difference $>$ 2.0 , while down-regulation is defined as having a fold difference $<0.5$.

\section{Western blot}

Cells were grown to $60-80 \%$ confluence and conditioned media/lysates were harvested. Conditioned media was spun down at $4^{\circ} \mathrm{C}$ using $10 \mathrm{~K}$ Amicon centrifugal filter (Millipore, Bedford, MA, USA) to concentrate the secreted protein. Cells were lysed using RIPA buffer and Western blot analyses were performed as previously described [17]. Antibodies used in this study are summarized in Supplementary Table S1. Quantification of the bands was performed using ImageJ software (National Institutes of Health, Bethesda, MD USA) [66]. 


\section{Colony formation assay}

The $2 \mathrm{D}$ and $3 \mathrm{D}$ colony formation assays were performed as described by Kan et al. [35] and Wong et al. [67], respectively. For 3D colony formation assay, the 12-well plate was coated with one layer of Matrigel (BD Biosciences, San Jose, CA, USA). Transduced cells $\left(2 \times 10^{4}\right)$ were seeded onto the Matrigel and allowed to grow for a week until tumor spheres became apparent. Ten images of different microscopic views at 100× magnification were captured and tumor spheres with diameters above $100 \mu \mathrm{m}$ were counted.

\section{Wound healing assay}

The wound healing assay was carried out as reported previously [65]. Wound closure percentage was calculated using the formula: (migrated distance at 24 hour $\div$ initial wound width $\times 100$. For relative migration ability, the percentage wound closure of NID2-expressing cells was normalized to that of vector control.

\section{Transwell migration and invasion assays}

The transwell assays were performed as described previously $[35,56]$. Briefly, $2 \times 10^{5}$ cells were seeded in serum-free medium in the top chamber of the BD BioCoat migration insert or Matrigel invasion insert (BD Biosciences, St Jose, CA, USA), fitted with an $8 \mu \mathrm{m}$ pore membrane. Culture medium enriched with $10 \%$ serum was used as chemoattractant in the bottom chamber. At the end of assay (24-48 hours), migrated and invaded cells were fixed and stained with crystal violet. Five different microscopic fields (100× magnification) of the chambers were taken and quantification was with ImageJ software (NIH, Bethesda, MD USA).

\section{Human phospho-kinase antibody array}

The protein lysates from the vector-alone (VA) and NID2-expressing HONE1 cells were used for the Human Phospho-Kinase Antibody Array (R\&D Systems, Minneapolis, MN, USA), as described [25]. Quantification of the array data was performed using ImageJ software (NIH, Bethesda, MD USA) [66].

\section{Cell proliferation assay}

MTT (3-[4,5-dimethylthiazol-2-yl]-2,5 diphenyl tetrazolium bromide) assay was utilized for the in vitro cell proliferation assay as previously described [56]. A total of $2 \times 10^{4}$ cells was seeded in 96-well plates and absorbance of MTT was measured daily for five consecutive days at $570 \mathrm{~nm}$.

\section{In vivo tumorigenicity and metastasis assays}

In vivo nude mouse subcutaneous injection was performed as described previously [57]. Briefly, $1 \times 10^{7}$ and $1 \times 10^{6}$ cells of transduced HONE1 and KYSE30 cells, respectively, were inoculated subcutaneously into the flanks of female BALB/cAnN-nu (nude) mice. Tumor growth was monitored weekly with the use of digital calipers.

Nude mouse intrasplenic injection was conducted for the in vivo metastasis assay as described [25]. A total of $1 \times 10^{6}$ cells of pLVX-EF $1 \alpha(\mathrm{VA}) / \mathrm{pLVX}-\mathrm{NID} 2$ transduced HONE1-Luc was injected into the spleen of the mice following laparotomy. Mice were monitored weekly by detecting the bioluminescent signals upon intraperitoneal injection of D-luciferin as described [25]. Live bioluminescent imaging was performed using the IVIS Spectrum In vivo Imaging System (PerkinElmer, Norwalk, CT, USA), available at the Core Facility of Li Ka Shing Faculty of Medicine, The University of Hong Kong. At the third week post-inoculation, mice were sacrificed and necropsy was performed. The livers of the mice were excised and examined for presence of metastasized tumors. All the animal experiments were conducted with valid license from the Department of Health, Hong Kong S.A.R. and approved by the Committee on the Use of Live Animals in Teaching and Research (CULATR) of The University of Hong Kong.

\section{Paraffin embedding and Hematoxylin and Eosin (H\&E) staining}

Excised tissues were paraffin embedded and H\&E stained as described [25].

\section{Statistical analysis}

Chi-square test or Fisher's exact test (if any cell size was less than 5) was used in the MS-HRM analysis of biopsies and the in vivo metastasis assay. Student's $t$-test was utilized for statistical analyses for other experiments unless stated otherwise. $P$-values below 0.05 were considered statistically significant.

\section{ACKNOWLEDGMENTS}

We thank Dr. Judy Yam for kindly providing the luciferase plasmid [68] and Dr. T Sasaki for kindly providing the NID2 ORF clone [5]. We acknowledge the Faculty Core Facility of the Li Ka Shing Faculty of Medicine, HKU for providing the in vivo animal imaging system. We also thank the NPC Area of Excellence (AoE) Tissue Bank for providing NPC tissue sections for IHC staining. 


\section{CONFLICTS OF INTEREST}

The authors declare no conflicts of interest.

\section{GRANT SUPPORT}

This work was supported by the Research Grants Council of the Hong Kong Special Administrative Region, People's Republic of China: General Research Fund grant number 17115214 to MLL.

\section{REFERENCES}

1. Jones PA, Baylin SB. The epigenomics of cancer. Cell. 2007; 128:683-692.

2. Herman JG, Merlo A, Mao L, Lapidus RG, Issa JPJ, Davidson NE, Sidransky D, Baylin SB. Inactivation of the Cdkn2/P16/Mts1 Gene Is Frequently Associated with Aberrant DNA Methylation in All Common Human Cancers. Cancer Res. 1995; 55:4525-4530.

3. Jones PA, Baylin SB. The fundamental role of epigenetic events in cancer. Nature reviews Genetics. 2002; 3:415-428.

4. Baylin SB, Herman JG, Graff JR, Vertino PM, Issa JP. Alterations in DNA methylation: a fundamental aspect of neoplasia. Adv Cancer Res. 1998; 72:141-196.

5. Kohfeldt E, Sasaki T, Gohring W, Timpl R. Nidogen-2: A new basement membrane protein with diverse binding properties. J Mol Biol. 1998; 282:99-109.

6. Miosge N, Holzhausen S, Zelent C, Sprysch P, Herken R. Nidogen-1 and nidogen-2 are found in basement membranes during human embryonic development. Histochem J. 2001; 33:523-530.

7. Ulazzi L, Sabbioni S, Miotto E, Veronese A, AngustiA, GafaR, Manfredini S, Farinati F, Sasaki T, Lanza G, Negrini M. Nidogen 1 and 2 gene promoters are aberrantly methylated in human gastrointestinal cancer. Mol Cancer. 2007; 6 .

8. Renard I, Joniau S, van Cleynenbreugel B, Collette C, Naome C, Vlassenbroeck I, Nicolas H, de Leval J, Straub J, Van Criekinge W, Hamida W, Hellel M, Thomas A, et al. Identification and validation of the methylated TWIST1 and NID2 genes through real-time methylation-specific polymerase chain reaction assays for the noninvasive detection of primary bladder cancer in urine samples. European urology. 2010; 58:96-104.

9. Yegin Z, Gunes S, Buyukalpelli R. Hypermethylation of TWIST1 and NID2 in Tumor Tissues and Voided Urine in Urinary Bladder Cancer Patients. DNA Cell Biol. 2013; 32:386-392.

10. Gustafson KS, Davis AR, Van Neste L, Ronnett BM, Herman JG. Discovery of Novel Methylated Genes in Cervical Carcinogenesis: Methylation of GREM1 and NID2 is Detected in the Majority of Invasive Cervical Cancers and Defines a Subset of High-Grade Squamous Intraepithelial Lesions. Cancer Cytopathol. 2009; 117:351-351.
11. Esteller M, Sanchez-Cespedes M, Rosell R, Sidransky D, Baylin SB, Herman JG. Detection of aberrant promoter hypermethylation of tumor suppressor genes in serum DNA from non-small cell lung cancer patients. Cancer Res. 1999; 59:67-70.

12. Karnes RJ, Fernandez CA, Shuber AP. A noninvasive multianalyte urine-based diagnostic assay for urothelial cancer of the bladder in the evaluation of hematuria. Mayo Clinic proceedings. 2012; 87:835-842.

13. Abern MR, Owusu R, Inman BA. Clinical performance and utility of a DNA methylation urine test for bladder cancer. Urologic oncology. 2014; 32:51 e21-56.

14. Guerrero-Preston R, Soudry E, Acero J, Orera M, MorenoLopez L, Macia-Colon G, Jaffe A, Berdasco M, IliGangas C, Brebi-Mieville P, Fu Y, Engstrom C, IrizarryRA, et al. NID2 and HOXA9 Promoter Hypermethylation as Biomarkers for Prevention and Early Detection in Oral Cavity Squamous Cell Carcinoma Tissues and Saliva. Cancer Prev Res. 2011; 4:1061-1072.

15. Mokkapati S, Bechtel M, Reibetanz M, Miosge N, NischtR. Absence of the Basement Membrane Component Nidogen 2, but Not of Nidogen 1, Results in Increased Lung Metastasis in Mice. J Histochem Cytochem. 2012; 60:280-289.

16. Dai W, Cheung AK, Ko JM, Cheng Y, Zheng $\mathrm{H}$, Ngan RK, Ng WT, Lee AW, Yau CC, Lee VH, Lung ML. Comparative methylome analysis in solid tumors reveals aberrant methylation at chromosome $6 \mathrm{p}$ in nasopharyngeal carcinoma. Cancer medicine. 2015.

17. Cheung AKL, Lung HL, Hung SC, Law EWL, Cheng Y, Yau WL, Bangarusamy DK, Miller LD, Liu ETB, Shao JY, Kou CW, Chua D, Zabarovsky ER, et al. Functional analysis of a cell cycle-associated, tumor-suppressive gene, protein tyrosine phosphatase receptor type $\mathrm{G}$, in nasopharyngeal carcinoma. Cancer Res. 2008; 68:8137-8145.

18. Cheung AK, Lung HL, Ko JM, Cheng Y, Stanbridge EJ, Zabarovsky ER, Nicholls JM, Chua D, Tsao SW, Guan XY, Lung ML. Chromosome 14 transfer and functional studies identify a candidate tumor suppressor gene, mirror image polydactyly 1 , in nasopharyngeal carcinoma. Proceedings of the National Academy of Sciences of the United States of America. 2009; 106:14478-14483.

19. Lung HL, Bangarusamy DK, Xie D, Cheung AKL, Cheng Y, Kumaran MK, Miller L, Liu ETB, Guan XY, Sham JS, Fang Y, Li LQ, Wang N, et al. THY1 is a candidate tumour suppressor gene with decreased expression in metastatic nasopharyngeal carcinoma. Oncogene. 2005; 24:6525-6532.

20. Lo PHY, Xie D, Chan KC, Xu FP, Kuzmin I, Lerman MI, Law S, Chua D, Sham J, Lung ML. Reduced expression of RASSF1A in esophageal and nasopharyngeal carcinomas significantly correlates with tumor stage. Cancer Lett. 2007; 257:199-205.

21. Law EW, Cheung AK, Kashuba VI, Pavlova TV, Zabarovsky ER, Lung HL, Cheng Y, Chua D, Lai-Wan Kwong D, Tsao SW, Sasaki T, Stanbridge EJ, Lung ML. Anti-angiogenic and tumor-suppressive roles of candidate 
tumor-suppressor gene, Fibulin-2, in nasopharyngeal carcinoma. Oncogene. 2012; 31:728-738.

22. Chen H, Ko JM, Wong VC, Hyytiainen M, Keski-Oja J, Chua D, Nicholls JM, Cheung FM, Lee AW, Kwong DL, Chiu PM, Zabarovsky ER, Tsao SW, et al. LTBP-2 confers pleiotropic suppression and promotes dormancy in a growth factor permissive microenvironment in nasopharyngeal carcinoma. Cancer Lett. 2012; 325:89-98.

23. Lung HL, Cheung AKL, Ko JMY, Cheng Y, Stanbridge EJ, Lung ML. Deciphering the molecular genetic basis of NPC through functional approaches. Semin Cancer Biol. 2012; 22:87-95.

24. Dai W, Zheng H, Cheung AK, Lung ML. Genetic and epigenetic landscape of nasopharyngeal carcinoma. Chinese clinical oncology. 2016; 5:16.

25. Cheung AK, Ip JC, Chu AC, Cheng Y, Leong MM, KoJM, Shuen WH, Lung HL, Lung ML. PTPRG suppresses tumor growth and invasion via inhibition of Akt signaling in nasopharyngeal carcinoma. Oncotarget. 2015; 6:13434-13447. doi: 10.18632/oncotarget.3876.

26. Lo KW, Teo PM, Hui AB, To KF, Tsang YS, Chan SY, Mak KF, Lee JC, Huang DP. High resolution allelotype of microdissected primary nasopharyngeal carcinoma. Cancer Res. 2000; 60:3348-3353.

27. Ko JM, Yau WL, Chan PL, Lung HL, Yang L, Lo PH, TangJC, Srivastava G, Stanbridge EJ, Lung ML. Functional evidence of decreased tumorigenicity associated with monochromosome transfer of chromosome 14 in esophageal cancer and the mapping of tumor-suppressive regions to 14q32. Genes, chromosomes \& cancer. 2005; 43:284-293.

28. Reinert T, Modin C, Castano FM, Lamy P, Wojdacz TK, Hansen LL, Wiuf C, Borre M, Dyrskjot L, Orntoft TF. Comprehensive genome methylation analysis in bladder cancer: identification and validation of novel methylated genes and application of these as urinary tumor markers. Clinical cancer research. 2011; 17:5582-5592.

29. Geng JF, Sun JF, Lin Q, Gu J, Zhao YX, Zhang HY, FengX, He YH, Wang W, Zhou XY, Yu J. Methylation status of NEUROG2 and NID2 improves the diagnosis of stage I NSCLC. Oncol Lett. 2012; 3:901-906.

30. Maldonado L, Brait M, Michailidi C, Munari E, DriscollT, Schultz L, Bivalacqua T, Schoenberg M, SidranskyD, Netto GJ, Hoque MO. An epigenetic marker panel for recurrence risk prediction of low grade papillary urothelial cell carcinoma (LGPUCC) and its potential use for surveillance after transurethral resection using urine. Oncotarget. 2014; 5:5218-5233. doi: 10.18632/ oncotarget.2129.

31. Lu P, Weaver VM, Werb Z. The extracellular matrix: a dynamic niche in cancer progression. The Journal of cell biology. 2012; 196:395-406.

32. Kim SH, Turnbull J, Guimond S. Extracellular matrix and cell signalling: the dynamic cooperation of integrin, proteoglycan and growth factor receptor. The Journal of endocrinology. 2011; 209:139-151.
33. Lukashev ME, Werb Z. ECM signalling: orchestrating cell behaviour and misbehaviour. Trends Cell Biol. 1998; $8: 437-441$

34. Chan SHK, Ko JMY, Chan KW, Chan YP, Tao Q, Hyytiainen M, Keski-Oja J, Law S, Srivastava G, Tang J, Tsao SW, Chen H, Stanbridge EJ, et al. The ECM protein LTBP-2 is a suppressor of esophageal squamous cell carcinoma tumor formation but higher tumor expression associates with poor patient outcome. Int J Cancer. 2011; 129:565-573.

35. Kan R, Shuen WH, Lung HL, Cheung AK, Dai W, KwongDL, Ng WT, Lee AW, Yau CC, Ngan RK, TungSY, Lung ML. NF-kappaB p65 Subunit Is Modulated by Latent Transforming Growth Factor-beta Binding Protein 2 (LTBP2) in Nasopharyngeal Carcinoma HONE1 and HK1 Cells. Plos One. 2015; 10:e127239.

36. Lo PH, Lung HL, Cheung AK, Apte SS, Chan KW, KwongFM, Ko JM, Cheng Y, Law S, Srivastava G, Zabarovsky ER, Tsao SW, Tang JC, et al. Extracellular protease ADAMTS9 suppresses esophageal and nasopharyngeal carcinoma tumor formation by inhibiting angiogenesis. Cancer Res. 2010; 70:5567-5576.

37. Lung HL, Lo PHY, Xie D, Apte SS, Cheung AKL, ChengY, Law EWL, Chua D, Zeng YX, Tsao SW, Stanbridge EJ, Lung ML. Characterization of a novel epigeneticallysilenced, growth-suppressive gene, ADAMTS9, and its association with lymph node metastases in nasopharyngeal carcinoma. Int J Cancer. 2008; 123:401-408.

38. Chan KC, Ko JMY, Lung HL, Sedlacek R, Zhang ZF, Luo DZ, Feng ZB, Chen S, Chen HL, Chan KW, TsaoSW, Chua DTT, Zabarovsky ER, et al. Catalytic activity of matrix metalloproteinase-19 is essential for tumor suppressor and anti-angiogenic activities in nasopharyngeal carcinoma. Int J Cancer. 2011; 129:1826-1837.

39. Valastyan S, Weinberg RA. Tumor Metastasis: Molecular Insights and Evolving Paradigms. Cell. 2011; 147:275-292.

40. Franken NAP, Rodermond HM, Stap J, Haveman J, van Bree C. Clonogenic assay of cells in vitro. Nat Protoc. 2006; 1:2315-2319.

41. Seewaldt V. ECM stiffness paves the way for tumor cells. Nature medicine. 2014; 20:332-333.

42. Mouw JK, Yui Y, Damiano L, Bainer RO, Lakins JN, AcerbiI, Ou GQ, Wijekoon AC, Levental KR, Gilbert PM, Hwang ES, Chen YY, Weaver VM. Tissue mechanics modulate microRNA-dependent PTEN expression to regulate malignant progression. Nature medicine. 2014; 20:360-+.

43. Mitra SK, Schlaepfer DD. Integrin-regulated FAK-Src signaling in normal and cancer cells. Current opinion in cell biology. 2006; 18:516-523.

44. Aoudjit F, Vuori K. Integrin signaling in cancer cell survival and chemoresistance. Chemotherapy research and practice. 2012; 2012:283181.

45. Yurchenco PD. Basement Membranes: Cell Scaffoldings and Signaling Platforms. Csh Perspect Biol. 2011; 3. 
46. Ilic D, Almeida EA, Schlaepfer DD, Dazin P, AizawaS, Damsky CH. Extracellular matrix survival signals transduced by focal adhesion kinase suppress p53-mediated apoptosis. The Journal of cell biology. 1998; 143:547-560.

47. Hood JD, Cheresh DA. Role of integrins in cell invasion and migration. Nature reviews Cancer. 2002; 2:91-100.

48. Yip WK, Leong VC, Abdullah MA, Yusoff S, Seow HF. Overexpression of phospho-Akt correlates with phosphorylation of EGF receptor, FKHR and BAD in nasopharyngeal carcinoma. Oncology reports. 2008; 19:319-328.

49. Lin DC, Hao JJ, Nagata Y, Xu L, Shang L, Meng X, Sato Y, Okuno Y, Varela AM, Ding LW, Garg M, Liu LZ, Yang H, et al. Genomic and molecular characterization of esophageal squamous cell carcinoma. Nat Genet. 2014; 46:467-473.

50. Hanawa M, Suzuki S, Dobashi Y, Yamane T, Kono K, Enomoto N, Ooi A. EGFR protein overexpression and gene amplification in squamous cell carcinomas of the esophagus. Int J Cancer. 2006; 118:1173-1180.

51. Bill HM, Knudsen B, Moores SL, Muthuswamy SK, Rao VR, Brugge JS, Miranti CK. Epidermal growth factor receptor-dependent regulation of integrin-mediated signaling and cell cycle entry in epithelial cells. Molecular and cellular biology. 2004; 24:8586-8599.

52. Cabodi S, Moro L, Bergatto E, Boeri Erba E, Di StefanoP, Turco E, Tarone G, Defilippi P. Integrin regulation of epidermal growth factor (EGF) receptor and of EGFdependent responses. Biochem Soc Trans. 2004; 32:438-442.

53. Huang DP, Ho JH, Poon YF, Chew EC, Saw D, Lui M, LiCL, Mak LS, Lai SH, Lau WH. Establishment of a cell line (NPC/HK1) from a differentiated squamous carcinoma of the nasopharynx. Int J Cancer. 1980; 26:127-132.

54. Cheung ST, Huang DP, Hui AB, Lo KW, Ko CW, Tsang YS, Wong N, Whitney BM, Lee JC. Nasopharyngeal carcinoma cell line (C666-1) consistently harbouring Epstein-Barr virus. Int J Cancer. 1999; 83:121-126.

55. Li HM, Man C, Jin Y, Deng W, Yip YL, Feng HC, CheungYC, Lo KW, Meltzer PS, Wu ZG, Kwong YL, YuenAP, Tsao SW. Molecular and cytogenetic changes involved in the immortalization of nasopharyngeal epithelial cells by telomerase. Int J Cancer. 2006; 119:1567-1576.

56. Lung HL, Cheung AKL, Cheng Y, Kwong FM, Lo PHY, Law EWL, Chua D, Zabarovsky ER, Wang N, Tsao SW, Stanbridge EJ, Lung ML. Functional characterization of THY 1 as a tumor suppressor gene with antiinvasive activity in nasopharyngeal carcinoma. Int J Cancer. 2010; 127:304-312.

57. Cheng Y, Poulos NE, Lung ML, Hampton G, Ou BX, Lerman MI, Stanbridge EJ. Functional evidence for a nasopharyngeal carcinoma tumor suppressor gene that maps at chromosome 3p21.3. Proceedings of the National Academy of Sciences of the United States of America. 1998; 95:3042-3047.

58. Yu VZ, Wong VCL, Dai W, Ko JMY, Lam AKY, ChanKW, Samant RS, Lung HL, Shuen WH, Law S, Chan YP,
LeeNPY, Tong DKH, et al. Nuclear Localization of DNAJB6 Is Associated With Survival of Patients With Esophageal Cancer and Reduces AKT Signaling and Proliferation of Cancer Cells. Gastroenterology. 2015; 149:1825-+.

59. Wong VC, Chen H, Ko JM, Chan KW, Chan YP, Law S, Chua D, Kwong DL, Lung HL, Srivastava G, Tang JC, Tsao SW, Zabarovsky ER, et al. Tumor suppressor dualspecificity phosphatase 6 (DUSP6) impairs cell invasion and epithelial-mesenchymal transition (EMT)-associated phenotype. Int J Cancer. 2012; 130:83-95.

60. Yang X, Dai W, Kwong DL, Szeto CY, Wong EH, Ng WT, Lee AW, Ngan RK, Yau CC, Tung SY, Lung ML. Epigenetic markers for noninvasive early detection of nasopharyngeal carcinoma by methylation-sensitive high resolution melting. Int J Cancer. 2015; 136:E127-135.

61. Lo PH, Leung AC, Kwok CY, Cheung WS, Ko JM, Yang LC, Law S, Wang LD, Li J, Stanbridge EJ, Srivastava G, Tang JC, Tsao SW, et al. Identification of a tumor suppressive critical region mapping to $3 \mathrm{p} 14.2$ in esophageal squamous cell carcinoma and studies of a candidate tumor suppressor gene, ADAMTS9. Oncogene. 2007; 26:148-157.

62. Ko JM, Chan PL, Yau WL, Chan HK, Chan KC, YuZY, Kwong FM, Miller LD, Liu ET, Yang LC, Lo PH, Stanbridge EJ, Tang JC, et al. Monochromosome transfer and microarray analysis identify a critical tumor-suppressive region mapping to chromosome 13q14 and THSD1 in esophageal carcinoma. Molecular cancer research. 2008; 6:592-603.

63. LC L, R D. MethPrimer: designing primers for methylation PCRs. . Bioinformatics. 2002; 18:1427-1431.

64. Wojdacz TK, Hansen LL, Dobrovic A. A new approach to primer design for the control of PCR bias in methylation studies. BMC research notes. 2008; 1:54.

65. Phoon YP, Cheung AK, Cheung FM, Chan KF, Wong S, Wong BW, Tung SY, Yau CC, Ng WT, Lung ML. IKBB tumor suppressive role in nasopharyngeal carcinoma via NF-kappaB-mediated signalling. Int J Cancer. 2015.

66. Schneider CA, Rasband WS, Eliceiri KW. NIH Image to ImageJ: 25 years of image analysis. Nature methods. 2012; 9:671-675.

67. Wong VCL, Chan PL, Bernabeu C, Law S, Wang LD, Li JL, Tsao SW, Srivastava G, Lung ML. Identification of an invasion and tumor-suppressing gene, Endoglin (ENG), silenced by both epigenetic inactivation and allelic loss in esophageal squamous cell carcinoma. Int J Cancer. 2008; 123:2816-2823.

68. Tse EYT, Ko FCF, Tung EKK, Chan LK, Lee TKW, Ngan ESW, Man K, Wong AST, Ng IOL, Yam JWP. Caveolin-1 overexpression is associated with hepatocellular carcinoma tumourigenesis and metastasis. Journal of Pathology. 2012; 226:645-653. 